\title{
The RAB39B p.G192R mutation causes X-linked dominant Parkinson's disease
}

\author{
Ignacio F. Mata ${ }^{1,2 \dagger}$, Yongwoo Jang ${ }^{3 \dagger}$, Chun-Hyung Kim³ ${ }^{3}$, David S. Hanna ${ }^{4,5}$, Michael O. Dorschner ${ }^{4,5}$, Ali Samii ${ }^{1,2}$, \\ Pinky Agarwal ${ }^{6}$, John W. Roberts ${ }^{7}$, Olga Klepitskaya ${ }^{8}$, David R. Shprecher ${ }^{9}$, Kathryn A. Chung ${ }^{10,11}$, Stewart A. Factor ${ }^{12}$, \\ Alberto J. Espay ${ }^{13}$, Fredy J. Revilla ${ }^{14}$, Donald S. Higgins ${ }^{15}$, Irene Litvan ${ }^{16}$, James B. Leverenz ${ }^{17}$, Dora Yearout ${ }^{1,2}$, \\ Miguel Inca-Martinez ${ }^{18}$, Erica Martinez ${ }^{1,2}$, Tiffany R. Thompson ${ }^{1}$, Brenna A. Cholerton ${ }^{1,4}$, Shu-Ching Hu ${ }^{1,2}$, \\ Karen L. Edwards ${ }^{19}$, Kwang-Soo Kim ${ }^{3}$ and Cyrus P. Zabetian ${ }^{1,2^{*}}$
}

\begin{abstract}
Objective: To identify the causal gene in a multi-incident U.S. kindred with Parkinson's disease (PD).

Methods: We characterized a family with a classical PD phenotype in which 7 individuals ( 5 males and 2 females) were affected with a mean age at onset of 46.1 years (range, 29-57 years). We performed whole exome sequencing on 4 affected and 1 unaffected family members. Sanger-sequencing was then used to verify and genotype all candidate variants in the remainder of the pedigree. Cultured cells transfected with wild-type or mutant constructs were used to characterize proteins of interest.

Results: We identified a missense mutation (c.574G > A; p.G192R) in the RAB39B gene that closely segregated with disease and exhibited $X$-linked dominant inheritance with reduced penetrance in females. The mutation occurred in a highly conserved amino acid residue and was not observed among 87,725 X chromosomes in the Exome Aggregation Consortium dataset. Sequencing of the $R A B 39 B$ coding region in 587 familial PD cases yielded two additional mutations (c.428C > G [p.A143G] and c.624_626delGAG [p.R209del]) that were predicted to be deleterious in silico but occurred in families that were not sufficiently informative to assess segregation with disease. Experiments in PC12 and SK-N-BE(2)C cells demonstrated that p.G192R resulted in mislocalization of the mutant protein, possibly by altering the structure of the hypervariable C-terminal domain which mediates intracellular targeting.

Conclusions: Our findings implicate RAB39B, an essential regulator of vesicular-trafficking, in clinically typical PD. Further characterization of normal and aberrant RAB39B function might elucidate important mechanisms underlying neurodegeneration in PD and related disorders.
\end{abstract}

\section{Background}

Parkinson's disease (PD) is the second most common neurodegenerative disorder and though approximately $20 \%$ of patients report a family history of the disease, kindreds that display clear Mendelian inheritance are rare. However, mutations in several genes have been shown to result in clinically typical autosomal dominant (SNCA, LRRK2, VPS35, DNAJC13) or recessive (PARK2, PINK1, PARK7) PD, or parkinsonism with atypical

\footnotetext{
*Correspondence: zabetian@u.washington.edu

${ }^{\dagger}$ Equal contributors

'Veterans Affairs Puget Sound Health Care System, Seattle, WA, USA

${ }^{2}$ Department of Neurology, University of Washington School of Medicine,

Seattle, WA, USA

Full list of author information is available at the end of the article
}

features (e.g. PARK9) [1]. Functional characterization of these genetic variants has provided important insights into the molecular mechanisms underlying $\mathrm{PD}$ and elucidated novel targets for therapeutic intervention.

Whole-exome sequencing (WES) is a powerful tool for gene discovery in pedigrees that are not sufficiently large for traditional linkage analysis [2] and this technique has been successful in identifying two causal genes for PD $[3,4]$. In this study we present data from WES and in vitro functional analyses that demonstrate that a missense mutation (p.G192R) in the $R A B 39 B$ gene is the causative variant in a multi-incident family with clinically typical PD.

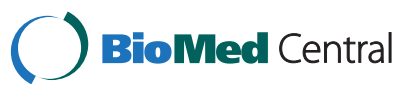

(C) 2015 Mata et al. Open Access This article is distributed under the terms of the Creative Commons Attribution 4.0 International License (http://creativecommons.org/licenses/by/4.0/), which permits unrestricted use, distribution, and reproduction in any medium, provided you give appropriate credit to the original author(s) and the source, provide a link to the Creative Commons license, and indicate if changes were made. The Creative Commons Public Domain Dedication waiver (http://creativecommons.org/publicdomain/zero/1.0/) applies to the data made available in this article, unless otherwise stated. 


\section{Results}

We studied a U.S. family of European origin in which 7 individuals ( 5 males and 2 females) were affected and met UK PD Society Brain Bank clinical diagnostic criteria for PD [5] (Fig. 1a). The clinical characteristics of the affected family members are provided in Table 1 . None of these individuals displayed atypical findings on neurological examination but two of them (III-15 and IV-4) had mild intellectual disability since childhood. DNA was available for 6 affected and 10 unaffected members of the family. We performed WES on 4 affected (III-4, III-9, III-11, and III-18) and 1 unaffected (III-13) family members. We filtered out all variants with a frequency $>1 \%$ in 515 controls from the NHLBI Exome Sequencing Project $[6,7]$ or that failed to meet the quality thresholds of the Genome Analysis ToolKit (GATK) "Best Practices" [8]. We identified three nonsynonymous variants that passed all filters, segregated with PD among the 5 individuals who underwent WES, and were confirmed by Sanger sequencing: USP1 c.573G > A (p.M191I), MVP c.2594G > T (p.G865V), and RAB39B c.574G > A (p.G192R) (Table 2). We then genotyped these three variants in all remaining family members and found that only RAB39B p.G192R was present in all six affected subjects. Furthermore,
RAB39B p.G192R was not observed among $87,725 \mathrm{X}$ chromosomes successfully sequenced for $R A B 39 B$ in the Exome Aggregation Consortium database (ExAC; http://exac.broadinstitute.org). The amino acid G192 is highly conserved across species (Fig. 1b) and this mutation is predicted to be deleterious as evidenced by a Combined Annotation Dependent Depletion (CADD) [9] score of 29.4.

We then screened for $R A B 39 B$ p.G192R in 2 cohorts of PD patients from the Parkinson's Genetic Research Study (PaGeR). Cohort I was comprised of 203 "multiplex" families ascertained from across the U.S. (mean age at onset of probands, 57.3 years; male, $61.9 \%$ ) in which at least 2 individuals with PD were enrolled. Cohort II included 1298 unrelated PD patients (mean age at onset, 59.1 years; male, $69.2 \%$ ) enrolled primarily at movement disorder clinics in the Pacific Northwest regardless of family history. $R A B 39 B$ p.G192R was not found in any other PD patients across PaGeR cohorts I and II (total $\mathrm{n}=1501$ ). We also sequenced the entire $R A B 39 B$ coding region in the subset of patients from both PaGeR cohorts $(n=587)$ who reported a family history of PD and discovered two additional mutations, c. $428 \mathrm{C}>\mathrm{G}$ (p.A143G) and c.624_626delGAG (p.R209del). Neither

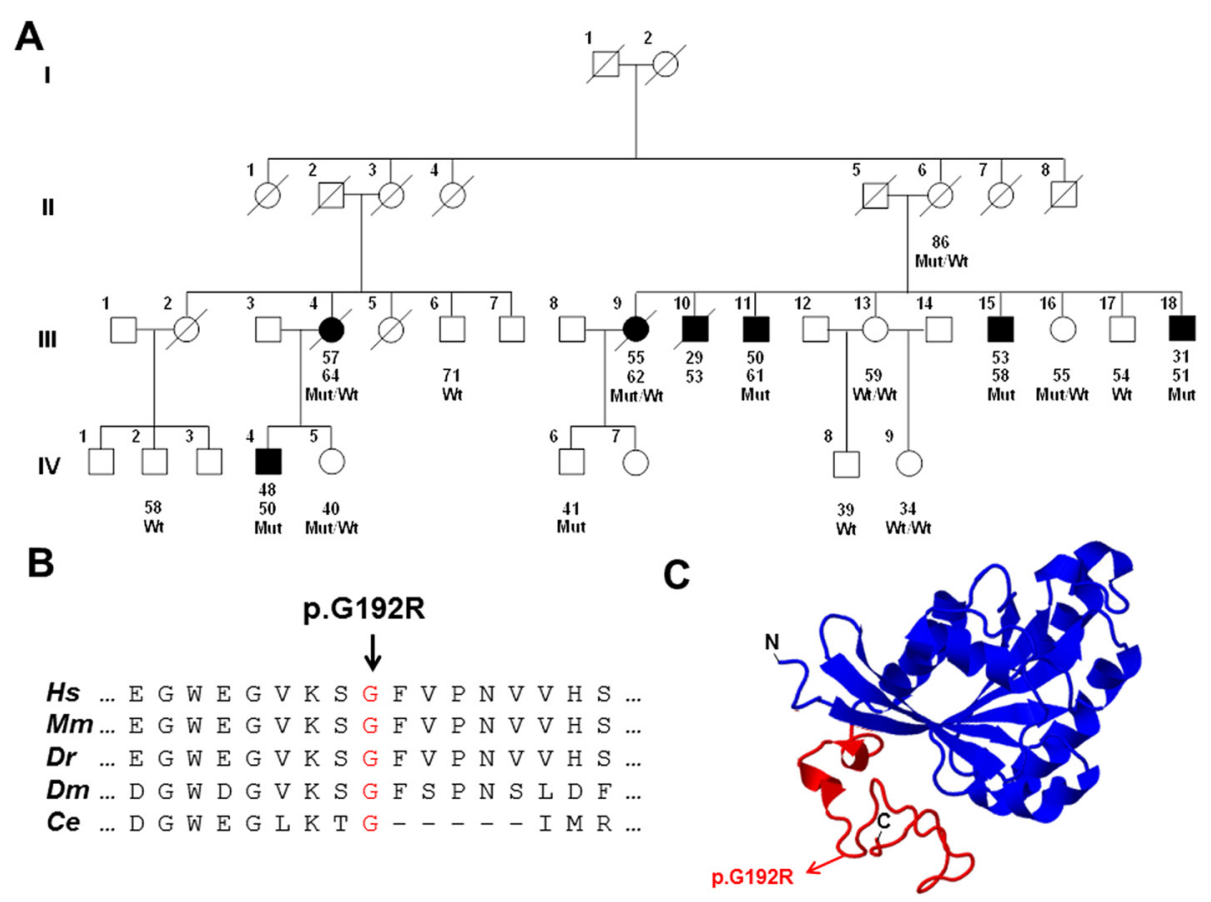

Fig. 1 Identification of the RAB39B p.G192R mutation by whole exome sequencing in a multigenerational kindred with Parkinson's disease. a Pedigree diagram; individuals affected with Parkinson's disease are represented with black symbols, unaffected individuals with open symbols. Age at onset is indicated immediately below each symbol, followed by age at last clinical evaluation. Wt = wild type; Mut = mutation (p.G192R). b Multispecies protein sequence alignment. Hs= Homo sapiens; Mm = Mus musculus; Dr= Danio rerio (zebrafish); Dm = Drosophila melanogaster; $\mathrm{Ce}=$ Caenorhabditis elegans. $\mathbf{c}$ Predicted protein structure of RAB39B using Protein Homology/analogY Recognition Engine V 2.0 (Phyre ${ }^{2}$; http:// www.sbg.bio.ic.ac.uk/phyre2/html/page.cgi?id=index) [23]; $89 \%$ of the amino acid residues were modeled at $>90 \%$ confidence. P.G192R is located in the hypervariable C-terminal domain which is depicted in red 
Table 1 Clinical characteristics of the affected members of the pedigree

\begin{tabular}{|c|c|c|c|c|c|c|c|}
\hline \multirow[t]{2}{*}{ Characteristic } & \multicolumn{7}{|c|}{ Patient } \\
\hline & $|I|-4$ & || $\mid-9$ & $|I| \mid-10$ & $|I| \mid-11$ & $|I| \mid-15$ & || $\mid-18$ & $\overline{I V-4}$ \\
\hline Sex & $\mathrm{F}$ & $\mathrm{F}$ & M & M & M & M & M \\
\hline Age at onset, yr & 57 & 55 & 29 & 50 & 53 & 31 & 48 \\
\hline Age at last evaluation, yr & 64 & 62 & 53 & 61 & 58 & 51 & 50 \\
\hline Age at death, yr & 70 & 64 & 56 & - & - & - & - \\
\hline Bradykinesia & Y & Y & Y & Y & Y & Y & Y \\
\hline Rigidity & Y & Y & Y & Y & Y & Y & Y \\
\hline Resting tremor & Y & N & Y & Y & Y & Y & Y \\
\hline Postural instability & Y & $\mathrm{N}$ & Y & Y & N & Y & Y \\
\hline Unilateral onset & Y & Y & Y & Y & Y & Y & Y \\
\hline Levodopa response & Y & Y & Y & Y & Y & Y & NT \\
\hline Levodopa-induced dyskinesia & N & Y & Y & Y & N & Y & - \\
\hline Mild, lifelong intellectual disability & $\mathrm{N}$ & $\mathrm{N}$ & $\mathrm{N}$ & $\mathrm{N}$ & Y & $\mathrm{N}$ & Y \\
\hline Hoehn and Yahr stage ${ }^{a}$ & 4 & 3 & 4 & 2.5 & 2 & 5 & 2.5 \\
\hline MDS-UPDRS III score ${ }^{a}$ & NA & 24 & NA & 26 & 19 & 52 & 11 \\
\hline
\end{tabular}

MDS-UPDRS III = Movement Disorder Society Unified Parkinson's Disease Rating Scale Part III; NA = not available; NT = no trial ${ }^{\text {a }}$ Determined at last evaluation

of these mutations were present in the ExAC dataset and both are predicted to be deleterious in silico with CADD scores of 21.8 (p.A143G) and 20.4 (p.R209del). Each mutation was present in a single family but neither of the two pedigrees was sufficiently informative to assess segregation with disease (Additional file 1: Figure S1).

We then investigated the effects of $R A B 39 B$ p.G192R in vitro. In PC12 and SK-N-BE(2)C cells transfected with mutant and wild-type constructs there was no substantial difference in RAB39B protein expression (Figs. 2a and 3a). In NGF-differentiated PC12 cells wild-type RAB39B protein was visualized throughout the cytoplasm of cell bodies and neuritic processes (Fig. 2b), and co-localized with the vesicular marker chromogranin A. However, mutant (p.G192R) RAB39B was largely restricted to cell bodies with negligible amounts of protein evident in neuritic processes. In these experiments there was robust expression of both mutant and wild type RAB39B protein, but cellular phenotype can sometimes differ based on the level of transgene over-expression [10].
Thus we used an alternate vector and method of transfection to over-express $\mathrm{RAB} 39 \mathrm{~B}$ at lower levels in retinoic acid-differentiated SK-N-BE(2)C cells. Wild type RAB39B protein was frequently visualized within the cytoplasm and at the plasma membrane (co-localized with EGFR; Fig. 3b). However, while mutant RAB39B protein was also abundant in the cytoplasm, it was less frequently observed at the plasma membrane. To quantify these findings we performed immunoblot analysis of fractionated protein extracts from these cells (Fig. 3c). The proportion of membrane-bound to cytosolic RAB39B protein was significantly lower in cells expressing mutant protein than wild type protein $(p<0.01)$.

\section{Discussion}

In the present study we provide strong evidence that the missense mutation p.G192R in $R A B 39 B$ results in clinically typical, levodopa responsive $\mathrm{PD}$. The pattern of inheritance is X-linked dominant with reduced penetrance among females. Age at onset varied by nearly three decades and was lower in males. A single male mutation

Table 2 Candidate variants identified by whole exome sequencing

\begin{tabular}{|c|c|c|c|c|c|c|c|c|}
\hline \multirow[t]{2}{*}{ Gene } & \multirow[t]{2}{*}{ Position (hg19) } & \multirow[t]{2}{*}{ Transcript } & \multirow[t]{2}{*}{$\mathrm{dbSNP}$} & \multicolumn{2}{|l|}{ Variation } & \multirow{2}{*}{$\begin{array}{l}\text { Allele } \\
\text { frequency }(\%)^{a}\end{array}$} & \multirow[t]{2}{*}{ CADD score } & \multirow[t]{2}{*}{ Segregation $^{\mathrm{b}}$} \\
\hline & & & & Nucleotide & Amino acid & & & \\
\hline USP1 & Chr1:62910424 & NM_001017415.1 & - & $c .573 G>A$ & p.M191I & 0 & 27.1 & $5 / 6$ \\
\hline MVP & Chr16:29859222 & NM_005115.4 & rs151174471 & c. $2594 \mathrm{G}>\mathrm{T}$ & p.G865V & 0.07 & 7.19 & $5 / 6$ \\
\hline$R A B 39 B$ & ChrX:154490156 & NM_171998.2 & - & c. $574 G>A$ & p.G192R & 0 & 29.4 & $6 / 6$ \\
\hline
\end{tabular}

CADD Combined Annotation Dependent Depletion

${ }^{\text {a } F r e q u e n c y ~ a m o n g ~ c h r o m o s o m e s ~ s u c c e s s f u l l y ~ s e q u e n c e d ~ f o r ~ U S P 1 ~}(\mathrm{n}=111,418), M V P(\mathrm{n}=121,248)$, and $R A B 39 B(\mathrm{n}=87,725)$ in the Exome Aggregation Consortium database (http://exac.broadinstitute.org)

${ }^{\mathrm{b}}$ Number of affected individuals with the variant/total number of affected individuals with genotypes in the pedigree 


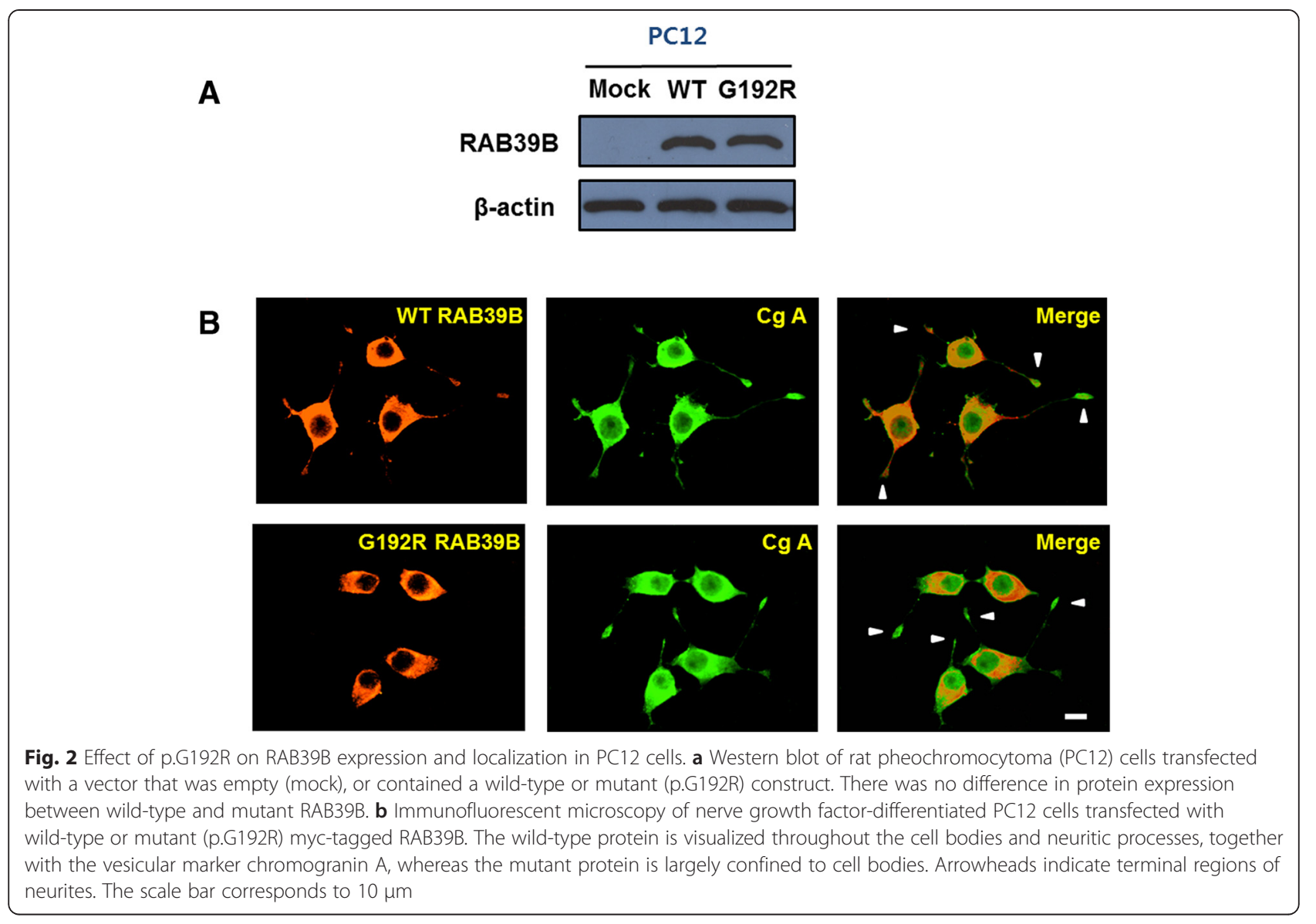

carrier was unaffected at age 41. Since his current age is below the age at onset observed for 3 of the 5 affected males in the pedigree, he might well become symptomatic over time. Alternatively, it is possible that the presence of other genetic or environmental factors are necessary for p.G192R to achieve full penetrance.

$R A B 39 B$ was first linked to human disease in 2010 when a nonsense and a splice site mutation in the gene were shown to cause mental retardation, sometimes accompanied by epilepsy and autism spectrum disorder, in the male members of two families [11]. Subsequently, duplications and triplications of a genomic region containing $R A B 39 B$ were discovered in males from three families with a similar phenotype [12]. Recently, two families were reported with early onset parkinsonism in males and a missense mutation (p.T168K) or a complete deletion of $R A B 39 B$ [13]. An autopsy of one such subject showed dopaminergic neuron loss in the substantia nigra and widespread Lewy body pathology. However, affected individuals all had features that were atypical for PD including intellectual disability, macrocephaly, and in the majority of cases, a lack of response to levodopa. In contrast, most individuals in our family displayed a classical PD phenotype, with the exception of two affected males who had mild intellectual disability, and both males and females were affected.

The explanation for the wide range of phenotypes associated with $R A B 39 B$ mutations is not entirely clear. Previously reported mutations result in either overexpression of wild-type protein [12] or a complete loss of protein expression [11, 13]. However, the mutation discovered in our family (p.G192R) did not substantially change the overall amount of RAB39B protein expressed in PC12 or SK-N-BE(2)C cells. Instead, p.G192R appears to alter intracellular localization as the mutant protein did not properly migrate to the neuritic processes of NGF-differentiated PC12 cells and to the plasma membrane in SK-N-BE(2)C cells. This is consistent with the structural location of p.G192R based on current knowledge of Rab proteins. RAB39B is one of over 60 members of the human Rab GTPase family [14]. Rab GTPases act as molecular switches, cycling between active (GTPbound) and inactive (GDP-bound) states to regulate intracellular vesicular trafficking in a temporally and spatially sensitive manner [15]. The C-terminus of Rab proteins contains a hypervariable domain (HVD) of 35-40 amino acids which through interactions with effector proteins plays a major role in targeting each Rab to the appropriate 


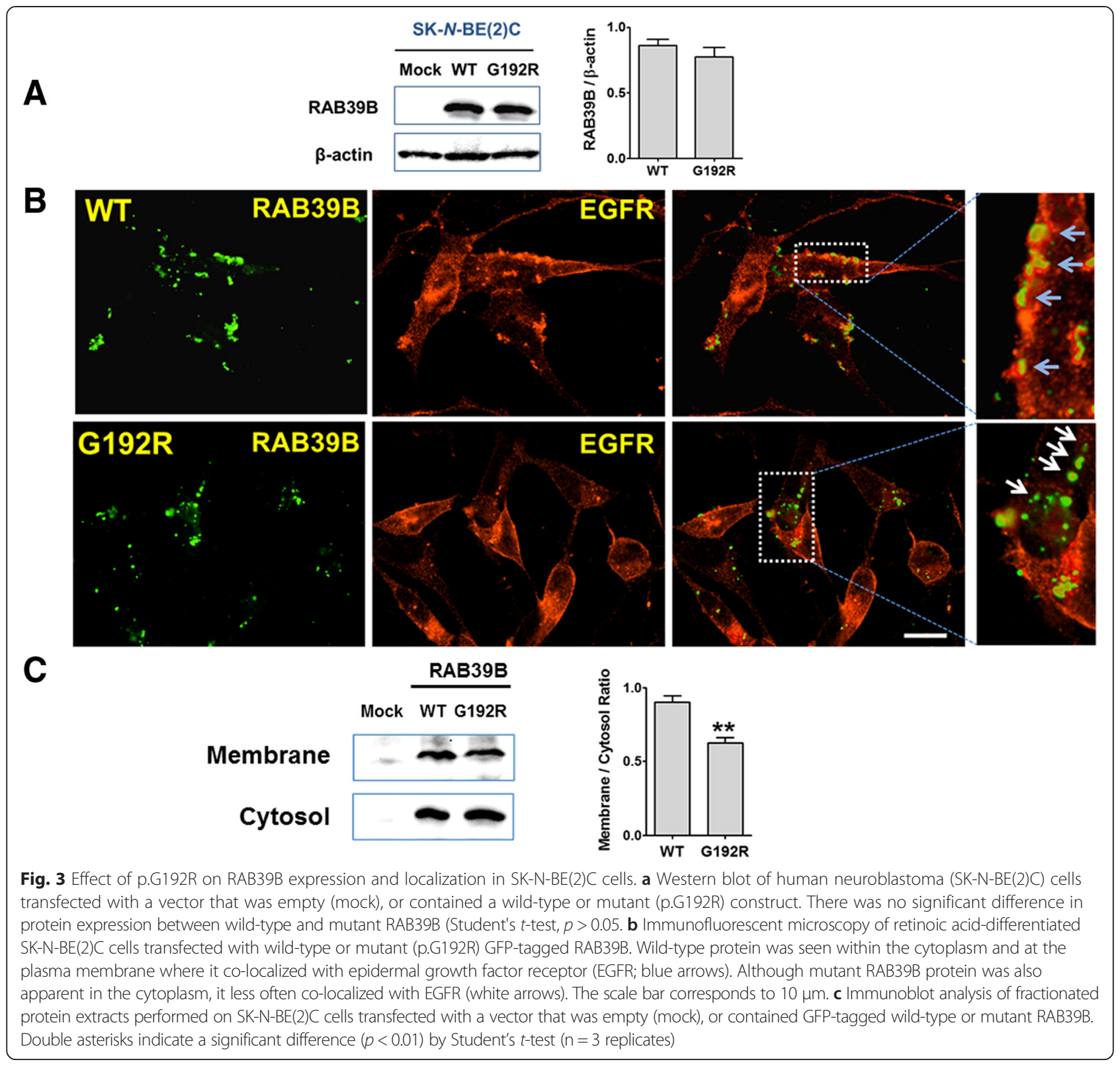

intracellular location [14]. The HVD has a high content of helix-breaking proline and glycine residues which contribute to the extended structure that allows for necessary protein interactions [16]. Because p.G192R is located within the HVD (Fig. 1c) and eliminates one such glycine moiety the mutation might disrupt proper targeting of RAB39B by inhibiting binding to effector molecules. Furthermore, the fact that two heterozygous females in our pedigree were affected raises the possibility that p.G192R might exert its pathogenic effects through a dominant negative mechanism. Interestingly, a dominant negative mutation in another Rab protein (Rab8) has been described that when introduced into Xenopus laevis induces retinal degeneration and shifts localization of the protein from Golgi and post-Golgi membranes to the cytoplasm [17].

Though many Rab proteins are well studied, the precise localization and function of $\mathrm{RAB} 39 \mathrm{~B}$ are just beginning to emerge. RAB39B is neuron-specific and plays a role in synapse formation and maintenance [11]. Recent evidence suggests that one function of RAB39B, through its interaction with protein interacting with C-kinase 1 (PICK1), is to regulate the subunit composition of heterotetrameric AMPA receptors [18]. In the absence of RAB39B, AMPA receptor composition shifts towards non GluA2-containing $\mathrm{Ca}_{2}^{+}$-permeable forms. The resulting alteration in synaptic activity has been posited to underlie the lifelong intellectual disability and 
behavioral problems seen with loss-of-function mutations in $R A B 39 B$. However, the mechanism by which dysregulation of $R A B 39 B$ leads to the selective neurodegenerative changes seen in PD is not yet known. Our discovery that p.G192R results in a "pure" PD phenotype provides an opportunity to address this important question by examining the effects of this mutation in model systems in future studies.

\section{Conclusions}

Our findings implicate RAB39B, an essential regulator of vesicular-trafficking, in clinically typical PD. Loss-offunction mutations in this gene were previously shown to cause X-linked recessive mental retardation sometimes accompanied by autism spectrum disorder, and members of two such families were later shown to develop atypical parkinsonism. However, the phenotype in our family is classical, levodopa-responsive PD and both males and females are affected. We present in vitro data that provide a potential explanation for the substantial difference in phenotype between our family and those reported elsewhere. Unlike previously reported mutations which result in a complete loss of protein expression, the mutation that we have discovered (p.G192R) does not alter the overall amount of protein expressed but rather its intracellular localization. Our results suggest that dysregulation of RAB39B, which is thought to mediate vesicular transport, can lead to selective neurodegenerative changes in the absence of lifelong cognitive/behavioral dysfunction, and have important implications for future research.

\section{Methods}

\section{Exome sequencing}

The exome was captured using the SeqCap EZ Exome v3.0 kit (Roche/Nimblegen, Madison, WI) and sequenced with 100-base pair (bp) paired-end reads on a HiSeq2500 (Illumina, San Diego, CA) to achieve a mean coverage of 80-100X. Sequence reads were mapped to the human reference genome (GRCh37) using the Burrows-Wheeler Aligner. Variants were jointly called using the GATK HaplotypeCaller following the developer's recommended best practices [8] (https://www.broadinstitute.org/gatk/ guide/best-practices) and annotated with SnpEff [19] based on the RefSeq gene set (http://www.ncbi.nlm.nih.gov/ refseq). We flagged variants failing to meet the quality thresholds described by the GATK "Best Practices" of QD (Quality by Depth) <2.0, FS (FisherStrand; Fisher's exact test for strand bias) >60.0, MQ (Mapping Quality; overall mapping quality of reads averaged over all samples) $<40.0$, HaplotypeScore (probability that reads flanking a variant can be explained by $\leq 2$ haplotypes) $>13.0$, MQRankSum (comparison of mapping qualities of reads for reference versus alternate allele) $<-12.5$, and ReadPosRankSum (measure of bias in position within reads between reference and alternate allele) $<-8.0$. We excluded alleles that occurred at a frequency $>1 \%$ in 515 unrelated white controls selected from the NHLBI Exome Sequencing Project [6, 7]. Finally, we used custom software to analyze variants that passed all filters to identify alleles that segregated with disease.

\section{Sanger sequencing and genotyping}

Sanger sequencing was used to confirm and genotype candidate variants in all available members of the pedigree. We also sequenced the entire $R A B 39 B$ coding region and intron-exon boundaries in the probands from PaGeR Cohort I $(n=203)$ and in the subset of patients from Cohort II $(\mathrm{n}=384)$ who reported a family history of PD. Sequencing was performed using the Applied Biosystems Big-Dye Terminator v3.1 Cycle Sequencing Kit on an ABI PRISM 3130 genetic analyzer (Applied Biosystems, Foster City, CA) as described elsewhere [20]. Sequence data were base-called, aligned, and scanned for variation using Mutation Surveyor (SoftGenetics, State College, PA). $R A B 39 B$ p.G192R was genotyped in the remainder of PaGeR Cohort II using a custom TaqMan assay.

\section{Protein expression assays}

Two myc-tagged constructs encoding either wild type or mutant (p.G192R) RAB39B protein were created using the vector pcDNA 3.1/myc-His (Invitrogen Life Technologies, Carlsbad, CA). Rat pheochromocytoma (PC12) and human neuroblastoma (SK-N-BE(2)C) cells were grown and transfected with wild type or mutant constructs using either a retrovirus (pCL Vector System, Orbigen, San Diego, CA) for PC12 cells or Lipofectamine (Invitrogen) for SK-N-BE(2)C cells using previously described methods [21]. After $24 \mathrm{~h}$ the cells were lysed, and the lysates were subjected to $10 \%$ sodium dodecyl sulfate-polyacrylamide gel electrophoresis and transferred to a polyvinylidene difluoride membrane (Bio-Rad Laboratories, Hercules, CA). The membranes were incubated with an anti-myc antibody (Roche Life Sciences, Branford, CT) and detection was achieved using the Novex ECL chemiluminescent substrate reagent kit (Invitrogen).

\section{Protein trafficking experiments}

PC12 cells were transfected with a wild type or mutant (p.G192R) myc-tagged $R A B 39 B$ construct as described in the previous section and differentiated with nerve growth factor (NGF) for 4 days. The cells were fixed in $4 \%$ paraformaldehyde and then incubated overnight at $4{ }^{\circ} \mathrm{C}$ with rabbit anti-myc and anti-chromogranin A antibodies. SK-N-BE(2)C cells were transfected with GFP-tagged wild type or mutant $R A B 39 B$ constructs (pEGFP-N1 vector; Clontech Laboratories, Mountain View, CA) using Lipofectamine. After retinoic acid-induced differentiation for 4 days the cells were fixed in $4 \%$ paraformaldehyde and 
incubated overnight at $4{ }^{\circ} \mathrm{C}$ with anti-epidermal growth factor receptor (anti-EGFR) antibody (a plasma membrane marker; Cell Signaling Technology, Danvers, MA) using methods described elsewhere [22]. Hoechst 33342 was used for counterstaining and confocal analysis was performed using an Olympus IX81 microscope.

\section{Subcellular fractionation}

SK-N-BE(2)C cells were transfected with GFP-tagged wild type or mutant $R A B 39 B$ constructs as described in the previous section. After $24 \mathrm{~h}$ the cells were lysed and membrane and cytoplasmic fractions were prepared using the Subcellular Protein Fractionation kit for Cultured Cells (Thermo Scientific, Rockford, IL) according to the manufacturer's recommendations. The fractionated lysates were subjected to $10 \%$ sodium dodecyl sulfate-polyacrylamide gel electrophoresis and transferred to a polyvinylidene difluoride membrane. The membranes were incubated with an anti-GFP antibody (Santa Cruz Biotechnology). Images of western blots were captured using the ChemiDoc XRS system (Bio-Rad Laboratories) and scanned films were quantified using Image J software (http://rsb.info.nih.gov/ij/).

\section{Additional file}

Additional file 1: Figure S1. Pedigrees with variants of unknown significance. Pedigrees in which the RAB39B (A) c.428C>G (p.A143G) and (B) c.624_626delGAG (p.R209del) variants were observed. Individuals affected with Parkinson's disease are represented with black symbols, unaffected individuals with open symbols. Age at onset is indicated immediately below each symbol, followed by age at last clinical evaluation. $\mathrm{Wt}=$ wild type; Mut = mutation. (PDF $35 \mathrm{~kb})$

\section{Competing interests}

The authors declare that they have no competing interests.

\section{Authors' contributions}

IFM and CPZ had full access to all of the data in the study and take responsibility for the integrity of the data and the accuracy of the data analysis. Study concept and design: IFM, CPZ. Acquisition of data: YJ, C-HK, DSH, MOD, AS, PA, JWR, OK, DRS, KAC, SAF, AJE, FJR, DSH, IL, JBL, DY, MI-M, EM, TT, BAC, S-CH, K-SK . Analysis and interpretation of data: IFM, YJ, C-HK, DSH, MOD, KLE, KLE, CPZ. Drafting of the manuscript: IFM, CPZ. Critical revision of the manuscript for important intellectual content: YJ, C-HK, DSH, MOD, AS, PA, JWR, OK, DRS, KAC, SAF, AJE, FJR, DSH, IL, JBL, DY, MI-M, EM, TT, BAC, S-CH, KLE, K-SK. All authors read and approved the final manuscript.

\section{Acknowledgments}

This work was supported by grants from the National Institutes of Health (P50 NS062684, R01 NS065070, R01 NS084869) and the Department of Veterans Affairs (1101BX000531), and by a gift from the Dolsen Foundation. We thanks all of the individuals who participated in the study and Drs. Jennifer Witt and Rosalind Chuang for assistance with patient recruitment.

\section{Author details}

${ }^{1}$ Veterans Affairs Puget Sound Health Care System, Seattle, WA, USA. ${ }^{2}$ Department of Neurology, University of Washington School of Medicine, Seattle, WA, USA. ${ }^{3}$ Molecular Neurobiology Laboratory, Department of Psychiatry and Program in Neuroscience, McLean Hospital/Harvard Medical School, Belmont, MA, USA. ${ }^{4}$ Department of Psychiatry and Behavioral Sciences, University of Washington, Seattle, WA, USA. ${ }^{5}$ Department of
Pathology, University of Washington, Seattle, WA, USA. ${ }^{6}$ Booth Gardner Parkinson's Care Center, Evergreen Hospital Medical Center, Kirkland, WA, USA. ${ }^{7}$ Virginia Mason Medical Center, Seattle, WA, USA. ${ }^{8}$ Department of Neurology, University of Colorado, Denver, USA. ${ }^{9}$ Department of Neurology, University of Utah, Salt Lake City, UT, USA. ${ }^{10}$ Parkinson's Disease Research, Education, and Clinical Center, Portland Veterans Affairs Medical Center, Portland, OR, USA. ${ }^{11}$ Department of Neurology, Oregon Health and Science University, Portland, OR, USA. ${ }^{12}$ Department of Neurology, Emory University School of Medicine, Atlanta, GA, USA. ${ }^{13}$ Department of Neurology and Rehabilitation Medicine, University of Cincinnati, Cincinnati, OH, USA. ${ }^{14}$ Division of Neurology at Greenville Health System and the University of South Carolina Medical School-Greenville, Greenville, SC, USA. ${ }^{15}$ Samuel Stratton Veterans Affairs Medical Center, Albany, NY, USA. ${ }^{16}$ Movement Disorder Center, Department of Neurosciences, University of California, San Diego, CA, USA. ${ }^{17}$ Lou Ruvo Center for Brain Health, Cleveland Clinic, Cleveland, OH, USA. ${ }^{18}$ Neurogenetics Research Center, Instituto Nacional de Ciencias Neurologicas, Lima, Peru. ${ }^{19}$ Department of Epidemiology, University of California, Irvine, CA, USA.

Received: 31 July 2015 Accepted: 17 September 2015

Published online: 24 September 2015

\section{References}

1. Trinh J, Farrer M. Advances in the genetics of Parkinson disease. Nat Rev Neurol. 2013;9(8):445-54.

2. Singleton $A B$. Exome sequencing: a transformative technology. Lancet Neurol. 2011;10(10):942-6.

3. Zimprich A, Benet-Pages A, Struhal W, Graf E, Eck SH, Offman MN, et al. A mutation in VPS35, encoding a subunit of the retromer complex, causes late-onset Parkinson disease. Am J Hum Genet. 2011;89(1):168-75.

4. Vilarino-Guell C, Rajput A, Milnerwood AJ, Shah B, Szu-Tu C, Trinh J, et al. DNAJC13 mutations in Parkinson disease. Hum Mol Genet. 2014;23(7):1794-801.

5. Gibb WR, Lees AJ. The relevance of the Lewy body to the pathogenesis of idiopathic Parkinson's disease. J Neurol Neurosurg Psychiatry. 1988;51(6):745-52

6. Weeke P, Mosley JD, Hanna D, Delaney JT, Shaffer C, Wells QS, et al. Exome sequencing implicates an increased burden of rare potassium channel variants in the risk of drug-induced long QT interval syndrome. J Am Coll Cardiol. 2014;63(14):1430-7.

7. Tennessen JA, Bigham AW, O'Connor TD, Fu W, Kenny EE, Gravel S, et al. Evolution and functional impact of rare coding variation from deep sequencing of human exomes. Science. 2012;337(6090):64-9.

8. Carson AR, Smith EN, Matsui H, Braekkan SK, Jepsen K, Hansen JB, et al. Effective filtering strategies to improve data quality from population-based whole exome sequencing studies. BMC Bioinformatics. 2014;15:125.

9. Kircher M, Witten DM, Jain P, O'Roak BJ, Cooper GM, Shendure J. A general framework for estimating the relative pathogenicity of human genetic variants. Nat Genet. 2014;46(3):310-5.

10. Ferreira JP, Peacock RW, Lawhorn IE, Wang CL. Modulating ectopic gene expression levels by using retroviral vectors equipped with synthetic promoters. Syst Synth Biol. 2011;5(3-4):131-8.

11. Giannandrea M, Bianchi V, Mignogna ML, Sirri A, Carrabino S, D'Elia E, et al. Mutations in the small GTPase gene RAB39B are responsible for $\mathrm{X}$-linked mental retardation associated with autism, epilepsy, and macrocephaly. Am J Hum Genet. 2010;86(2):185-95.

12. Vanmarsenille L, Giannandrea M, Fieremans N, Verbeeck J, Belet S, Raynaud M, et al. Increased dosage of RAB39B affects neuronal development and could explain the cognitive impairment in male patients with distal Xq28 copy number gains. Hum Mutat. 2014;35(3):377-83.

13. Wilson GR, Sim JC, MCLean C, Giannandrea M, Galea CA, Riseley JR, et al. Mutations in RAB39B cause X-linked intellectual disability and early-onset Parkinson disease with alpha-synuclein pathology. Am J Hum Genet. 2014;95(6):729-35.

14. Hutagalung AH, Novick PJ. Role of Rab GTPases in membrane traffic and cell physiology. Physiol Rev. 2011;91(1):119-49.

15. Schwartz SL, Cao C, Pylypenko O, Rak A, Wandinger-Ness A. Rab GTPases at a glance. J Cell Sci. 2007;120(Pt 22):3905-10.

16. Pfeffer SR. Structural clues to Rab GTPase functional diversity. J Biol Chem. 2005;280(16):15485-8.

17. Moritz OL, Tam BM, Hurd LL, Peranen J, Deretic D, Papermaster DS. Mutant rab8 Impairs docking and fusion of rhodopsin-bearing post-Golgi 
membranes and causes cell death of transgenic Xenopus rods. Mol Biol Cell. 2001;12(8):2341-51.

18. Mignogna ML, Giannandrea M, Gurgone A, Fanelli F, Raimondi F, Mapelli L, et al. The intellectual disability protein RAB39B selectively regulates GluA2 trafficking to determine synaptic AMPAR composition. Nat Commun. 2015;6:6504.

19. Cingolani P, Platts $A$, le Wang $L$, Coon $M$, Nguyen T, Wang $L$, et al. A program for annotating and predicting the effects of single nucleotide polymorphisms, SnpEff: SNPs in the genome of Drosophila melanogaster strain w1118; iso-2; iso-3. Fly (Austin). 2012;6(2):80-92.

20. Tsuang D, Leverenz JB, Lopez OL, Hamilton RL, Bennett DA, Schneider JA, et al. GBA mutations increase risk for Lewy body disease with and without Alzheimer disease pathology. Neurology. 2012;79(19):1944-50.

21. Kim CH, Leung A, Huh YH, Yang E, Kim DJ, Leblanc P, et al. Norepinephrine deficiency is caused by combined abnormal mRNA processing and defective protein trafficking of dopamine beta-hydroxylase. J Biol Chem. 2011;286(11):9196-204

22. Jang Y, Lee MH, Lee J, Jung J, Lee SH, Yang DJ, et al. TRPM2 mediates the lysophosphatidic acid-induced neurite retraction in the developing brain. Pflugers Arch. 2014;466(10):1987-98.

23. Kelley LA, Sternberg MJ. Protein structure prediction on the Web: a case study using the Phyre server. Nat Protoc. 2009;4(3):363-71.

\section{Submit your next manuscript to BioMed Central and take full advantage of:}

- Convenient online submission

- Thorough peer review

- No space constraints or color figure charges

- Immediate publication on acceptance

- Inclusion in PubMed, CAS, Scopus and Google Scholar

- Research which is freely available for redistribution 